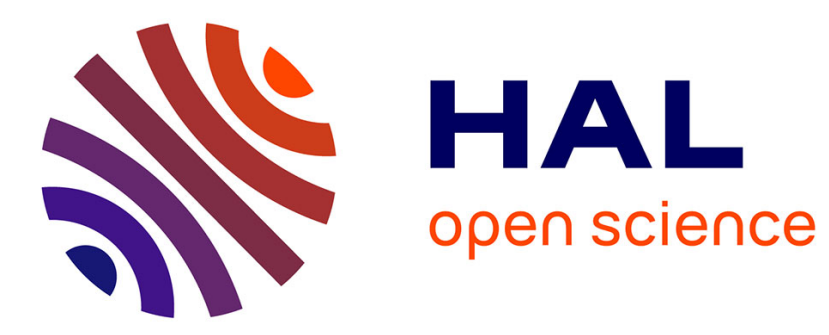

\title{
From Riemannian trichromacy to quantum color opponency via hyperbolicity
}

\author{
Michel Berthier, Edoardo Provenzi
}

\section{To cite this version:}

Michel Berthier, Edoardo Provenzi. From Riemannian trichromacy to quantum color opponency via hyperbolicity. 2020. hal-02479897

\section{HAL Id: hal-02479897 \\ https://hal.science/hal-02479897}

Preprint submitted on 14 Feb 2020

HAL is a multi-disciplinary open access archive for the deposit and dissemination of scientific research documents, whether they are published or not. The documents may come from teaching and research institutions in France or abroad, or from public or private research centers.
L'archive ouverte pluridisciplinaire HAL, est destinée au dépôt et à la diffusion de documents scientifiques de niveau recherche, publiés ou non, émanant des établissements d'enseignement et de recherche français ou étrangers, des laboratoires publics ou privés. 


\title{
From Riemannian trichromacy to quantum color opponency via hyperbolicity
}

\author{
Michel Berthier*1 and Edoardo Provenzi ${ }^{\dagger 2}$ \\ ${ }^{1}$ Laboratoire MIA, Université de La Rochelle, Avenue Albert Einstein, 17031 La \\ Rochelle BP 33060, France, Tel.: +33(0)5 46458605 \\ ${ }^{2}$ Université de Bordeaux, CNRS, Bordeaux INP, IMB, UMR 5251, F-33400, 351 \\ Cours de la Libération, Talence, France, Tel.: +33(0)5 40006437
}

\begin{abstract}
We propose a mathematical description of human color perception that relies on a hyperbolic structure of the space $\mathcal{P}$ of perceived colors. We show that hyperbolicity allows us to reconcile both trichromaticity, from a Riemannian point of view, and color opponency, from a quantum view-point. In particular, we will underline how the opponent behavior can be represented by a rebit, a real analog of a qubit, whose state space is endowed with the Hilbert metric.
\end{abstract}

\section{Introduction}

The modern description of color perception involves two complementary processes. The first one is based on the existence of the LMS cones with sensitivity pick in the long, medium and short wavelength range of the visible spectrum, respectively. This physiological fact is responsible for the trichromatic character of human color perception. The second process is related to the opponent color mechanism first described in 4 ] and then proven to be physiologically related to the action of retinal neurons, see e.g. [10].

Classically, the opponent mechanism is described as follows: for simplicity, let us denote again with $L, M, S$ the output of retinal cones, then the opposition $L-M$ or $M-L$ gives the red-green axis, the opposition $S-(L+M)$ or $(L+M)-S$ gives the blue-yellow axis, whereas the sum $L+M+S$, or the difference $-L-M-S$, gives the achromatic axis, i.e. the gray intensity. The Hering disk illustrates the oppositions by identifying $L-M$ with the point of the unit circle of angular coordinate $\theta=0, S$ with the point given by $\theta=\pi / 2, M-L$ with $\theta=\pi$ and $-S$ with $\theta=3 \pi / 2$. The center of the disk represents achromatic values.

Natural image statistics has systematically investigated the problem of why humans have evolved to develop such a peculiar codification system. To the best of our knowledge, the first description of chromatic opponency in this field has been provided by [6] via a principal component analysis (PCA) on a database of natural RGB images. The authors interpreted the ensemble of RGB image values as random variables and studied the covariance matrix of this statistical ensemble. If $v_{1}, v_{2}$ and $v_{3}$ are the eigenvectors of the covariance matrix relative to the eigenvalues $\lambda_{1}, \lambda_{2}$ and $\lambda_{3}$ such that $\left|\lambda_{1}\right|>\left|\lambda_{2}\right|>\left|\lambda_{3}\right|$, then their empirical results showed that $v_{1}$ is obtained via a linear combination of the $R, G, B$ axes with all positive coefficients, while $v_{2}$ and $v_{3}$ are built via linear combinations of $R, G$ and $B$ with coefficients of opposite sign. As a results, Ohta et al.'s PCA showed that the most efficient way to codify the chromatic content of the ensemble of RGB images was via the achromatic plus opponent channel representation foreseen by Hering.

\footnotetext{
*michel.berthier@univ-lr.fr

†edoardo.provenzi@math.u-bordeaux.fr
} 
The elegant work of $[3$ gave a rigorous explanation of the reason underlying the results just described. Due to the strong correlation between either the $L, M, S$ sensitivity curves, or the $R, G, B$ sensors of a camera, the chromatic covariance results in a positive matrix, i.e. a matrix with all positive coefficients, not to be confused with a positive-definite matrix. For positive matrices, the Perron-Frobenius theorem, see e.g. 2], guarantees that opponency must appear in the second and the third eigenvector.

[9] confirmed experimentally the predictions of Buchsbaum and Gottshalk by using a multispectral database. A more modern overview and further information about this topic can be found e.g. in [5] or [7].

It is nonetheless important to stress that what we have described above is a posteriori description of the phenomenon of chromatic opponency and not a mathematical model able to intrinsically account for this phenomenon. This remark applies also to the color description performed by the CIE color spaces as e.g. the CIELab space.

The main aim of this paper is to fill this gap by providing a novel model with respect to the state of the art in which color opponency appears naturally without the need of a posteriori analysis.

To do this, in section 2, we describe the possible Riemannian geometries of the space $\mathcal{P}$ of perceived colors viewed as a chromatic space. Classical historical axioms say that this chromatic space is a regular convex cone of dimension 3. Following the seminal work of $[8$ and under a supplementary axiom that is discussed in the sequel, we show that $\mathcal{P}$ must be isometric to either the flat space $\mathbb{R}^{+} \times \mathbb{R}^{+} \times \mathbb{R}^{+}$endowed with the Helmhotz-Stiles metric, or to the cone $\mathcal{H}^{+}(2, \mathbb{R})$ of positive definite $2 \times 2$ real symmetric matrices endowed with the Rao-Siegel metric. This latter is shown to be hyperbolic in the sense that it is foliated with leaves isometric to the Poincaré disk.

The fundamental remark that we develop in section 3 is that $\mathcal{H}(2, \mathbb{R})$, equipped with the Jordan matrix product, is the algebra of observables of a real quantum system with Hilbert space $\mathbb{R}^{2}$. This quantum system, called rebit, is a real analog of the qubit associated to $1 / 2$-spin particles. Then $\mathcal{H}^{+}(2, \mathbb{R})$ is the cone of a state space $\mathcal{S}$ that is isometric to the Klein disk, i.e. the unit disk equipped with the Hilbert metric.

As a consequence, we obtain the following mathematical description of human color perception: the perceived color space can be represented as the cone of positive elements of the Jordan algebra $\mathcal{H}(2, \mathbb{R})$ and it encodes both the Riemannian geometry of the trichromacy and the quantum opponent mechanism with the rebit, the Bloch disk of which coincides with the Hering disk. This proves that the missing mathematical link between the chromatic and color opponency stages is nothing but the hyperbolic structure of the space $\mathcal{P}$ of perceived colors.

We kept this paper intentionally short because we prefer to focus on the communication of the new ideas underlying our discoveries. All the many technical mathematical aspects will be detailed in future works.

\section{Riemannian trichromacy}

Our starting point is that the trichromatic space of perceived colors $\mathcal{P}$ is a regular convex cone of dimension 3. In order to classify the possible geometric structures of this space, in [8] a supplementary axiom is added. Starting from the observation that no color can be considered special with respect to another one, Resnikoff is led to postulate that $\mathcal{P}$ is a $G$-homogeneous space, where of course the group $G$ must represent the transformations on $\mathcal{P}$ that permit to pass from the perception of a color to that of any other color.

With a choice that is still debated today, Resnikoff selected for $G$ the following group: if $V=\operatorname{span}(\mathcal{P})$, then $G \equiv G L^{+}(\mathcal{P})=\{B \in G L(V): \operatorname{det}(B)>0, B x \in \mathcal{P} \forall x \in \mathcal{P}\}$. A transformation $B \in G L^{+}(\mathcal{P})$, i.e. an orientation-preserving linear map that preserves $\mathcal{P}$, is called a change of background illumination. Resnikoff noticed that by suitably changing the background illumination of a color stimulus, as in Fig. 1. we can modify its perception to any another color of $\mathcal{P}$ sufficiently close to it, i.e. $\mathcal{P}$ is a $G$-locally homogeneous space. Since $\mathcal{P}$ is a convex cone, 
local homogeneity implies that $G$ acts transitively on the whole $\mathcal{P}$, which then becomes a globally $G$-homogeneous cone.

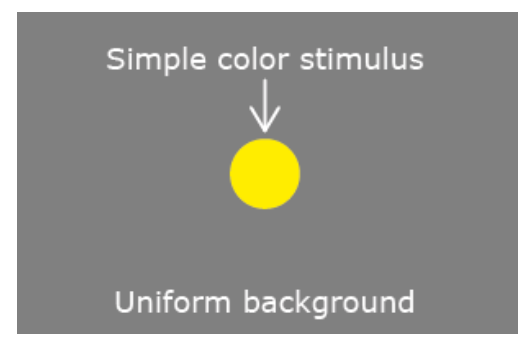

Figure 1: The observation arrangement considered by Resnikoff: a simple color stimulus, typically spanning a foveal aperture, embedded in a uniform background.

By using the dimension axiom, i.e. that $\operatorname{dim}(V) \leq 3$, it is possible to prove that $\mathcal{P}$ can be isomorphic to either the classical to colorimetric trichromatic space $\mathcal{P}_{1}=\mathbb{R}^{+} \times \mathbb{R}^{+} \times \mathbb{R}^{+}$or to a novel space for colorimetry, namely $\mathcal{P}_{2}=\mathcal{H}^{+}(2, \mathbb{R})$, the space of positive definite $2 \times 2$ real symmetric matrices.

Due to the essential role played by the group $G$ for his theory, Resnikoff searched for $G$ invariant Riemannian metrics on the spaces $\mathcal{P}_{1}$ and $\mathcal{P}_{2}$. It turned out that the only $G$-invariant Riemannian metric on the classical space $\mathcal{P}_{1}$ is the equally classical Helmholtz-Stiles metric $d s^{2}=$ $\alpha_{1}\left(\frac{d x_{1}}{x_{1}}\right)^{2}+\alpha_{2}\left(\frac{d x_{2}}{x_{2}}\right)^{2}+\alpha_{3}\left(\frac{d x_{3}}{x_{3}}\right)^{2}, \alpha_{i}>0, i=1,2,3$. Remarkably, the only $G$-invariant Riemannian metric on the space $\mathcal{P}_{2}$ is the Rao-Siegel metric $d s^{2}=\frac{1}{2} \operatorname{Trace}\left[\left(x^{-1} d x\right)^{2}\right]$.

If $\mathcal{H}_{1}^{+}(2, \mathbb{R})$ denotes the subset of matrices belonging to $\mathcal{H}^{+}(2, \mathbb{R})$ with unit determinant, then we have that $\mathcal{H}^{+}(2, \mathbb{R}) \simeq \mathbb{R}^{+} \times \mathcal{H}_{1}^{+}(2, \mathbb{R})$. One can check that, when endowed with the Rao-Siegel metric, this space is isometric to $\mathbb{R}^{+} \times \mathcal{D}$, where $\mathcal{D}$ is the Poincaré disk. An explicit isometry between the Poincaré disk $\mathcal{D}$ and $\mathcal{H}_{1}^{+}(2, \mathbb{R})$ is given by the following transformation:

$$
\begin{array}{ccc}
\varphi: \mathcal{D} & \longrightarrow \mathcal{H}_{1}^{+}(2, \mathbb{R}) \\
\left(w_{1}, w_{2}\right) & \longmapsto & \varphi\left(w_{1}, w_{2}\right)=\left(\begin{array}{cc}
\frac{1+2 w_{1}+\left(w_{1}^{2}+w_{2}^{2}\right)}{1-\left(w_{1}^{2}+w_{2}^{2}\right)} & \frac{2 w_{2}}{1-\left(w_{1}^{2}+w_{2}^{2}\right)} \\
\frac{2 w_{2}}{1-\left(w_{1}^{2}+w_{2}^{2}\right)} & \frac{1-2 w_{1}+\left(w_{1}^{2}+w_{2}^{2}\right)}{1-\left(w_{1}^{2}+w_{2}^{2}\right)}
\end{array}\right),
\end{array}
$$

so that we have:

$$
\frac{1}{2} \operatorname{Trace}\left[\left[\left(\varphi\left(w_{1}, w_{2}\right)\right)^{-1}\left(d \varphi\left(w_{1}, w_{2}\right)\right)\right]^{2}\right]=4\left(\frac{\left(d w_{1}\right)^{2}+\left(d w_{2}\right)^{2}}{\left(1-\left(w_{1}^{2}+w_{2}^{2}\right)^{2}\right.}\right)=d s_{\mathcal{D}}^{2} .
$$

At this stage, it is not clear how to recover the colorimetric attributes of perceived colors nor to give a meaningful description of the color opponency mechanism. In fact, it turns out that the missing link to obtain these results is given by Jordan algebras formalism. This not only permits to reformulate Resnikoff's description of $\mathcal{P}$ in a much more concise way, but, more importantly, it also opens a whole new perspective about color perception, formalizing a link with the algebraic theory of quantum physics.

According to the classification theorem of Jordan, von Neumann and Wigner, see e.g. 1, a formally real Jordan algebra of real dimension 3 is isomorphic to either $\mathbb{R} \oplus \mathbb{R} \oplus \mathbb{R}$ or $\mathcal{H}(2, \mathbb{R})$. The positive elements of these two Jordan algebras are the symmetric cones $\mathbb{R}^{+} \times \mathbb{R}^{+} \times \mathbb{R}^{+}$and $\mathcal{H}^{+}(2, \mathbb{R})$. This fact leads us to postulate only the following axiom for describing human color perception from a mathematical point of a view.

The trichromatic axiom: the space $\mathcal{P}$ of perceived colors is the symmetric cone of positive elements of a formally real Jordan algebra of real dimension 3.

Remark: we notice that, since symmetric cones are intrinsically homogeneous, this axiom has the advantage of circumventing the need of an explicit specification of a transitive action on $\mathcal{P}$, which is the most debated and controversial issue of Resnikoff's theory. 
The main contribution of this paper is to show that, contrary to the flat space $\mathcal{P}_{1}=\mathbb{R}^{+} \times$ $\mathbb{R}^{+} \times \mathbb{R}^{+}$, the hyperbolic space $\mathcal{P}_{2}=\mathcal{H}^{+}(2, \mathbb{R})$ is suitable to describe the dynamic of the chromatic opponent mechanism. One simple, but non trivial, result asserts that $\mathcal{H}(2, \mathbb{R})$ is isomorphic, as a Jordan algebra, to the spin factor $\mathbb{R} \oplus \mathbb{R}^{2}$ equipped with the Jordan product $\circ$ define by:

$$
(\alpha+u) \circ(\beta+v)=(\langle u, v\rangle+\alpha \beta+\alpha v+\beta u), \quad \forall \alpha, \beta \in \mathbb{R}, u, v \in \mathbb{R}^{2} .
$$

The explicit isomorphism of Jordan algebras between $\mathbb{R} \oplus \mathbb{R}^{2}$ and $\mathcal{H}(2, \mathbb{R})$ is given by:

$$
(\alpha+u) \longmapsto\left(\begin{array}{cc}
\alpha+u_{1} & u_{2} \\
u_{2} & \alpha-u_{1}
\end{array}\right) .
$$

If we consider the Minkowski metric induced by:

$$
(\alpha+v) \cdot(\beta+w)=\alpha \beta-\langle v, w\rangle,
$$

on $\mathbb{R} \oplus \mathbb{R}^{2}$, the hyperbolic space $\mathcal{H}^{+}(2, \mathbb{R})$ space corresponds isometrically to the future lightcone:

$$
\mathcal{L}^{+}=\{a=(\alpha+v), \alpha>0, a \cdot a>0\} .
$$

and $\mathcal{H}_{1}^{+}(2, \mathbb{R})$ to the level set:

$$
\mathcal{L}_{1}=\left\{a=(\alpha+v) \in \mathcal{L}^{+}, a \cdot a=1\right\} .
$$

This precisely means that $\mathcal{H}_{1}^{+}(2, \mathbb{R})$ equipped with the Rao-Siegel metric is isomorphic to the Klein disk

$$
\mathcal{K}=\left\{(\alpha+v) \in \overline{\mathcal{L}^{+}}, \alpha=1\right\},
$$

where $\overline{\mathcal{L}^{+}}$is the topological closure of $\mathcal{L}^{+}$, or to the Klein disk

$$
\mathcal{K}_{1 / 2}=\left\{(\alpha+v) \in \overline{\mathcal{L}^{+}}, \alpha=1 / 2\right\},
$$

with Klein metric given by the Hilbert metric. This latter disk provides a very simple vizualisation of the states of the quantum system described below. The fact that the distance on $\mathcal{K}_{1 / 2}$ is the Hilbert metric is a crucial fact that we will discuss in the conclusion.

\section{Quantum color opponency}

The algebra $\mathcal{H}(2, \mathbb{R})$ is the observable algebra of a real quantum system with Hilbert space $\mathbb{R}^{2}$. Such a system is called a rebit and it is a real analog of a qubit, i.e. a two-level complex quantum system of a $1 / 2$ spin particule. The state space $\mathcal{S}$ is the set of density matrices, i.e. the set of non-negative matrices of $\mathcal{H}(2, \mathbb{R})$ with trace equal to 1 . Such a density matrix can be written as:

$$
\rho\left(v_{1}, v_{2}\right)=\frac{1}{2}\left(\begin{array}{cc}
1+v_{1} & v_{2} \\
v_{2} & 1-v_{1}
\end{array}\right),
$$

where $v=\left(v_{1}, v_{2}\right) \in \mathbb{R}^{2}$ with $\|v\| \leq 1$. We can considere $\mathcal{S}$ as the unit disk $\left\{v \in \mathbb{R}^{2},\|v\| \leq 1\right\}$ embedded in the Klein disk $\mathcal{K}_{1 / 2}$ via the map

$$
s=\left(v_{1}, v_{2}\right) \longmapsto \frac{1}{2}(1+v)=\frac{1}{2}+\left(\frac{v_{1}}{2}, \frac{v_{2}}{2}\right) .
$$

Now, we explain the opponency mechanism of the rebit. First of all notice that, by direct computation, $\rho \in \mathcal{H}(2, \mathbb{R})$ is a state density matrix if and only if it can be written as:

$$
\rho\left(v_{1}, v_{2}\right)=\frac{1}{2}\left(I d_{2}+v \cdot \sigma\right)=\frac{1}{2}\left(I d_{2}+v_{1} \sigma_{1}+v_{2} \sigma_{2}\right),
$$


where $\sigma=\left(\sigma_{1}, \sigma_{2}\right)$ with:

$$
\sigma_{1}=\left(\begin{array}{cc}
1 & 0 \\
0 & -1
\end{array}\right), \quad \sigma_{2}=\left(\begin{array}{ll}
0 & 1 \\
1 & 0
\end{array}\right),
$$

and, again, $v=\left(v_{1}, v_{2}\right) \in \mathbb{R}^{2}$ with $\|v\| \leq 1$. The matrices $\sigma_{1}$ and $\sigma_{2}$ are Pauli-like matrices. They give a two direction opponency mechanism in the sens that we are going to explain.

By using the typical bra and ket Dirac notation of quantum mechanics, let us denote with $\left|u_{1}\right\rangle$, $\left|d_{1}\right\rangle,\left|u_{2}\right\rangle$ and $\left|d_{2}\right\rangle$ the four state vectors defined by:

$$
\begin{gathered}
\left|u_{1}\right\rangle=\left(\begin{array}{l}
1 \\
0
\end{array}\right),\left|d_{1}\right\rangle=\left(\begin{array}{l}
0 \\
1
\end{array}\right), \\
\left|u_{2}\right\rangle=\frac{1}{\sqrt{2}}\left(\begin{array}{l}
1 \\
1
\end{array}\right),\left|d_{2}\right\rangle=\frac{1}{\sqrt{2}}\left(\begin{array}{c}
-1 \\
1
\end{array}\right) .
\end{gathered}
$$

With a straightforward calculation, we can express $\sigma_{1}$ and $\sigma_{2}$ as a combination of projectors on the state vectors defined above, more precisely:

$$
\sigma_{1}=\left|u_{1}\right\rangle\left\langle u_{1}|-| d_{1}\right\rangle\left\langle d_{1}\left|, \sigma_{2}=\right| u_{2}\right\rangle\left\langle u_{2}|-| d_{2}\right\rangle\left\langle d_{2}\right| .
$$

The state vectors $\left|u_{1}\right\rangle$ and $\left|d_{1}\right\rangle$, resp. $\left|u_{2}\right\rangle$ and $\left|d_{2}\right\rangle$, are eigenstates of $\sigma_{1}$, resp. $\sigma_{2}$, with eigenvalues 1 and -1 . By using the polar coordinates reparameterization given by $v_{1}=r \cos \theta, v_{2}=r \sin \theta$, $r \in[0,1]$ and $\theta \in[0,2 \pi)$, we can write $\rho\left(v_{1}, v_{2}\right)$ as:

$$
\begin{aligned}
\rho(r, \theta)= & \frac{1}{2}\left(\begin{array}{cc}
1+r \cos \theta & r \sin \theta \\
r \sin \theta & 1-r \cos \theta
\end{array}\right) \\
= & \frac{1}{2}\left[(1+r \cos \theta)\left|u_{1}\right\rangle\left\langle u_{1}|+(1-r \cos \theta)| d_{1}\right\rangle\left\langle d_{1}\right|\right. \\
& \left.\quad+(r \sin \theta)\left|u_{2}\right\rangle\left\langle u_{2}|-(r \sin \theta)| d_{2}\right\rangle\left\langle d_{2}\right|\right] .
\end{aligned}
$$

This gives, for instanc $e^{1}$

$$
\begin{gathered}
L-M:=\rho(1,0)=\left|u_{1}\right\rangle\left\langle u_{1}\right|=\left(\begin{array}{cc}
1 & 0 \\
0 & 0
\end{array}\right), \\
M-L:=\rho(1, \pi)=\left|d_{1}\right\rangle\left\langle d_{1}\right|=\left(\begin{array}{cc}
0 & 0 \\
0 & 1
\end{array}\right), \\
S:=\rho(1, \pi / 2)=\left|u_{2}\right\rangle\left\langle u_{2}\right|=\frac{1}{2}\left(\begin{array}{cc}
1 & 1 \\
1 & 1
\end{array}\right), \\
-S:=\rho(1,3 \pi / 2)=\left|d_{2}\right\rangle\left\langle d_{2}\right|=\frac{1}{2}\left(\begin{array}{cc}
1 & -1 \\
-1 & 1
\end{array}\right) .
\end{gathered}
$$

More generally, for all $\theta \in[0,2 \pi)$, we have $\rho(1, \theta)=|(1, \theta)\rangle\langle(1, \theta)|$, with:

$$
|(1, \theta)\rangle=\cos (\theta / 2)\left|u_{1}\right\rangle+\sin (\theta / 2)\left|d_{1}\right\rangle .
$$

Let us recall that the pure state density matrices are state density matrices $\rho$ that are also projectors, i.e. which satisfy $\rho \circ \rho=\rho$. An equivalent characterization can be given in terms of the von Neumann entropy of the state density matrix $\rho$, i.e. $S(\rho)=-\operatorname{Trace}(\rho \log \rho): \rho$ defines a pure state if and only if $S(\rho)=0$.

The pure state density matrices correspond exactly to the points $(1+v) / 2$ of $\mathcal{K}_{1 / 2}$ with $\|v\|=1$, i.e. to the boundary points of $\mathcal{K}_{1 / 2}$. In fact, $\mathcal{K}_{1 / 2}$ is the Bloch disk of the rebit, a real analog of the Bloch ball of the qubit, embedded in $\overline{\mathcal{L}^{+}}$.

The opposite case is that of the maximal entropy state, that, for a general formally real Jordan algebra, corresponds to $\rho_{0} \equiv u / \operatorname{Trace}(u)$, where $u$ is its unit element. In particular, for the Jordan algebra $\mathcal{H}(2, \mathbb{R}), \rho_{0}=I d_{2} / 2$.

\footnotetext{
${ }^{1}$ We stress that the notations that we used here do not mean that $M-L$ is the opposite of $L-M$ and that $-S$ is the opposite of $S$. We adopt these notations according to the color opponency mechanism explained before.
} 


\subsection{Consequences on the mathematical description of color perception}

The previous mathematical discussion can be translated in colorimetric terms as follows. A perceived color $c=(\alpha+v)$ is an element of $\overline{\mathcal{L}^{+}}$, viewed as the Riemannian space of trichromacy. The real value $\alpha$ is the magnitude of the $c$. The element $c /(2 \alpha)=(1 / 2+v /(2 \alpha))$ belongs to the Klein disk, or the Bloch disk, $\mathcal{K}_{1 / 2}$. The chromatic state of $c$ is identified with the quantum state $s_{c}=v / \alpha$ of $\mathcal{S}$, the quantum chromatic state space.

A perceived color relative to a pure chromatic state, i.e. with zero von Neumann entropy, is a pure perceived color. On the contrary, a perceived color $c$ associated to a chromatic state with maximal von Neumann entropy, i.e. $\rho_{0}=I d_{2} / 2=(1+0) / 2$, is an achromatic perceived color. In fact, in the proposed framework, the classical attribute of saturation is nothing else than a mesure of the von Neumann entropy.

Consider now a chromatic state $s=\left(v_{1}, v_{2}\right)$ and the corresponding density matrix $\rho_{s}\left(v_{1}, v_{2}\right)=$ $\rho_{s}(r, \theta)$. In order to evaluate the chromatic state $s$ with regard to the opposition "red-green" given by $\sigma_{1}$, we compute the value:

$$
\left\langle\sigma_{1}\right\rangle_{\rho_{s}}=\operatorname{Trace}\left(\rho_{s} \circ \sigma_{1}\right)
$$

which gives the value of the observable $\sigma_{1}$ in the state $s$. We obtain:

$$
\left\langle\sigma_{1}\right\rangle_{\rho_{s}}=r \cos \theta .
$$

If $-\pi / 2<\theta<\pi / 2$, then $s$ is a reddish chromatic state, whereas it is greenish when $\pi / 2<\theta<$ $3 \pi / 2$. If $\theta=\pi / 2$ or $\theta=-\pi / 2$, then $s$ is achromatic in the opposition red-green. Note that Haring's statement: "greenness and redness can not be simultaneously attributes of a color" in this context is simply equivalent to the fact that $\cos \theta$ cannot be simultaneously non-negative and non-positive.

We obtain similar conclusions for the "yellow-blue" opposition given by $\sigma_{2}$ because:

$$
\left\langle\sigma_{2}\right\rangle_{\rho_{s}}=r \sin \theta .
$$

Finally, it easy to check that every density matrix $\rho(r, \theta)$ can be written as:

$$
\rho(r, \theta)=\rho_{0}+\frac{r \cos \theta}{2}(\rho(1,0)-\rho(1, \pi))+\frac{r \sin \theta}{2}(\rho(1, \pi / 2)-\rho(1,3 \pi / 2)),
$$

where the achromatic state density matrix is the mixture with equal probability:

$$
\rho_{0}=\frac{1}{4} \rho(1,0)+\frac{1}{4} \rho(1, \pi)+\frac{1}{4} \rho(1, \pi / 2)+\frac{1}{4} \rho(1,3 \pi / 2) .
$$

The difference $(\rho(1,0)-\rho(1, \pi))$ encodes the red-green opposition whereas the difference $(\rho(1, \pi / 2)-$ $\rho(1,3 \pi / 2))$ encodes the blue-yellow one.

To conclude, the quantum-like framework provided by the Jordan algebra formalism accounts for an intrinsic description of the achromatic plus opponent color phenomenon that does not require any a posteriori analysis.

\section{Conclusions and perspectives for future works}

We have shown that the human color perception can be mathematically described from the sole axiom that the trichromatic space is given by the positive elements of a Jordan algebra of real dimension 3. The hyperbolic Riemannian chromatic space is the future lightcone $\overline{\mathcal{L}^{+}}$of the 3 dimensional Minkoswki space. The Klein disk $\mathcal{K}_{1 / 2}$ of $\overline{\mathcal{L}^{+}}$appears to be the embedding of the chromatic quantum state space $\mathcal{S}$ of a rebit whose Bloch disk coincides with the Hering disk of color opponency.

One of the key points of this description of human color perception is that the hyperbolic Klein metric on $\mathcal{K}_{1 / 2}$ is given by the Hilbert distance. Hilbert's distance is defined on every convex set $\Omega$ and is always a Finsler metric whose asymmetric norm is given by:

$$
\|v\|_{p}=\frac{1}{2}\left(\frac{1}{\left|p-p^{-}\right|}+\frac{1}{\left|p-p^{+}\right|}\right)|v|
$$


where $p \in \Omega$ and $p^{ \pm}$are the intersection points with the boundary $\partial \Omega$ of the oriented line in $\Omega$ defined by the vector $v$ with Euclidean norm $|v|$ based at the point $p$. It is well known that this Finsler metric is Riemannian if and only if the boundary $\partial \Omega$ is an ellipse.

Now, both the Riemannian trichromatic and quantum chromatic spaces that we have described are ideal spaces that do not involve specific characteristics of an observer. In particular, the rebit is a so-called closed quantum system. It is natural to envisage to characterize every observer's capability regarding color perception by a convex subset $\Omega$ of the state space $\mathcal{S}$, or equivalently of the Klein disk $\mathcal{K}_{1 / 2}$, endowed with the Finsler metric given by the Hilbert distance. This convex subset $\Omega$ is in some sense the restriction of the ideal chromatic state space due to the limitation of the observer perception. Work in progress is devoted to identify, for each observer, the convex subset $\Omega$ by comparing the balls of the Finsler metric and the MacAdam ellipses drawn by the observer.

Finally, let us mention that the chromatic quantum system that we have described is, to the best of our knowledge, the first example of a meaningful real quantum system in nature, by opposition to the complex systems of quantum physics.

\section{References}

[1] John C Baez. Division algebras and quantum theory. Foundations of Physics, 42(7):819-855, 2012.

[2] A. Berman and R.J. Plemmons. Nonnegative Matrices in the Mathematical Sciences. SIAM, 1987.

[3] G. Buchsbaum and A. Gottschalk. Trichromacy, opponent colours coding and optimum colour information transmission in the retina. Proc. Royal Society of London B, 220:89-113, 1983.

[4] Ewald Hering. Zur lehre vom lichtsinne, volume 68. K. Akademie der Wissenschaften, 1878.

[5] A. Hyvärinen, J. Hurri, and P.O. Hoyer. Natural Image Statistics: A Probabilistic Approach to Early Computational Vision. Springer, 2009.

[6] Y. Ohta, T. Kanade, and T. Sakai. Color information for region segmentation. Computer graphics and image processing, 13:222-241, 1980.

[7] E. Provenzi, J. Delon, Y. Gousseau, and B. Mazin. On the second order spatiochromatic structure of natural images. Vision research, 120:22-38, 2016.

[8] H.L. Resnikoff. Differential geometry and color perception. Journal of Mathematical Biology, 1:97-131, 1974.

[9] D.L. Ruderman, T.W. Cronin, and C. Chiao. Statistics of cone responses to natural images: implications for visual coding. J. Opt. Soc. Am. A, 15(8):2036-2045, August 1998.

[10] S.K. Shevell and P.R. Martin. Color opponency: tutorial. JOSA A, 34(7):1099-1108, 2017. 